\title{
Human signatures derived from nighttime lights along the Eastern Alpine river network in Austria and Italy
}

\author{
Serena Ceola ${ }^{1}$, Alberto Montanari ${ }^{1}$, Juraj Parajka ${ }^{2}$, Alberto Viglione ${ }^{2}$, Günter Blöschl ${ }^{2,3}$, and \\ Francesco Laio ${ }^{4}$ \\ ${ }^{1}$ Dipartimento di Ingegneria Civile, Chimica, Ambientale e dei Materiali, Università di Bologna, \\ Bologna, 40136, Italy \\ ${ }^{2}$ Institute of Hydraulic Engineering and Water Resources Management, Vienna University of Technology, \\ Vienna, 1040, Austria \\ ${ }^{3}$ Centre for Water Resource Systems, Vienna University of Technology, Vienna, 1040, Austria \\ ${ }^{4}$ Dipartimento di Ingegneria dell'Ambiente, del Territorio e delle Infrastrutture, Politecnico di Torino,
} Torino, 10129, Italy

Correspondence to: Serena Ceola (serena.ceola@unibo.it)

Published: 12 May 2016

\begin{abstract}
Understanding how human settlements and economic activities are distributed with reference to the geographical location of streams and rivers is of fundamental relevance for several issues, such as flood risk management, drought management related to increased water demands by human population, fluvial ecosystem services, water pollution and water exploitation. Besides the spatial distribution, the evolution in time of the human presence constitutes an additional key question. This work aims at understanding and analysing the spatial and temporal evolution of human settlements and associated economic activity, derived from nighttime lights, in the Eastern Alpine region. Nightlights, available at a fine spatial resolution and for a 22-year period, constitute an excellent data base, which allows one to explore in details human signatures. In this experiment, nightlights are associated to five distinct distance-from-river classes. Our results clearly point out an overall enhancement of human presence across the considered distance classes during the last 22 years, though presenting some differences among the study regions. In particular, the river network delineation, by considering different groups of river pixels based on the Strahler order, is found to play a central role in the identification of nightlight spatiotemporal trends.
\end{abstract}

\section{Introduction}

Anthropogenic closeness to rivers is of fundamental relevance for socio-hydrological purposes, including flood and drought management, water pollution and exploitation, but also the human pressure on river ecosystems. In order to analyse human signatures in the proximity of streams and rivers, census data and satellite data such as Landscan could be easily employed (Small, 2004; Kummu et al., 2011), even though they cannot provide spatially and temporally detailed information about human presence. While census data are usually provided on a yearly basis at subnational level (i.e. coarse spatial resolution), spatially detailed Landscan data at $1 \mathrm{~km}$ resolution do not allow for a temporal trend analy- sis. To overcome this weakness, recent studies (Elvidge et al., 1997; Ceola et al., 2014, 2015) used fine scale remotely sensed data, as nighttime lights, to support global and local analyses of human presence and water related issues.

We explore here new opportunities offered by nightlight data to better decipher the interactions between human and water systems. More specifically, we provide insights about the spatio-temporal evolution of human presence along the river network in the European Eastern Alpine region and in its immediate proximity, by adopting satellite images of nighttime lights, available as yearly snapshots from 1992 to 2013 at a high spatial resolution (i.e., nearly $1 \mathrm{~km}$ at the equa- 
tor). In particular, nighttime lights are associated to the river network position and to additional four distance classes.

\section{Materials and methods}

\subsection{Satellite Nighttime Light Data Set}

Nighttime light time series, collected by the US Air Force Weather Agency under the Defense Meteorological Satellite Program (DMSP) - Operational Linescan System (OLS), are provided as freely available digital products by the National Geophysical Data Center from the National Oceanic and Atmospheric Administration (NOAA) at http://ngdc.noaa.gov/ eog/dmsp/downloadV4composites.html.

Nightlight data, produced on a yearly basis from 1992 to 2013, represent cloud-free nocturnal luminosity from sites with protracted lighting (i.e., cities, towns, gas flares). Sunlit and moonlit data and observations from ephemeral phenomena like fires are excluded from the data set. Nightlight values, expressed as an adimensional digital number $\mathrm{DN}_{\mathrm{obs}}$, range from 0 to 63 , corresponding to conditions characterized by absence of lights and pronounced luminosity, respectively. Nightlights thus represent a valuable proxy for the presence of human settlements and economic activity and they have been widely employed for demographic, economic, and environmental purposes (Elvidge et al., 1997, 2009; Small, 2004; Chand et al., 2009; Chen and Nordhaus, 2011; Bennie et al., 2014). Recently, Ceola et al. (2014, 2015) identified from nightlights data a higher human concentration in the vicinity of the river network, as well as a clear correlation between nightlights and economic losses due to flood events, showing that the more illuminated areas presented higher flood damages.

Nightlights cover almost the entire world $\left(180^{\circ} \mathrm{W}-180^{\circ} \mathrm{E}\right.$ longitude, $75^{\circ} \mathrm{N}-65^{\circ} \mathrm{S}$ latitude) and they are available as raster products at a very detailed spatial resolution, i.e., 30 arcsec, corresponding to nearly $1 \mathrm{~km}$ at the equator. Six different satellites collected the nighttime light data set, as reported in Table 1.

\subsection{River Network Data Set}

Regarding the location of the river network, the JRC's Catchment Characterisation and Modelling (CCM, version 2.1) open database is used (http://ccm.jrc.ec.europa.eu/php/index. php?action=view\&id=23). The CCM2 database covers the entire European continent, and it is based on a pan-European DEM mosaic with a 3 arcsec grid-cell resolution, updated land cover and climate data, and improved algorithms for data analysis.

\subsection{Study Region Administrative Boundaries Data Set}

In order to analyse the spatio-temporal evolution of human presence in the Eastern Alpine region, freely available vector files for Austria (http://www.naturalearthdata.com/ downloads/10m-cultural-vectors/10m-admin-0-countries/) and three North-Eastern Italian Regions (i.e., Trentino Alto Adige, Veneto and Friuli Venezia Giulia, http://www.istat.it/it/archivio/24613) are employed.

\subsection{Preparation of Repurposed Satellite Nightlight Data}

In order to get a unique and representative nightlight value for each pixel and for each year, for those years presenting two different satellites operating simultaneously (i.e., 1994, 1997, 1998, 1999, 2000, 2001, 2002, 2003, 2004, 2005, 2006, 2007, see Table 1), a new nightlight product is first obtained as the average nocturnal luminosity from the two overlapped data sets.

Second, because raw nightlight data are not on-board calibrated and cannot be compared among the 22 year period, a preliminary intercalibration procedure is required. Therefore, a well-established empirically based intercalibration technique is employed (see e.g., Elvidge et al., 2009; Chen and Nordhaus, 2011). This technique uses F121999 as the reference satellite composite, because it is characterized by the highest $\mathrm{DN}_{\mathrm{obs}}$ values that correspond exactly to the brightest areas of urban centres. The reference region for the intercalibration procedure is Sicily, since it presents a wide spread of $\mathrm{DN}_{\mathrm{obs}}$ values with a more sharply defined diagonal clusters of points, but also it does not show significant changes in nightlights over time. Thus, by applying the intercalibration procedure, satellite nightlight composites from Sicily are compared among years to find suitable regression coefficients by using:

$\mathrm{DN}=C_{0}+C_{1} \cdot \mathrm{DN}_{\mathrm{obs}}+C_{2} \cdot \mathrm{DN}_{\mathrm{obs}}^{2}$,

where DN refers to intercalibrated nightlight values, $\mathrm{DN}_{\mathrm{obs}}$ represents raw nightlight data and $C_{0}, C_{1}$ and $C_{2}$ are the empirical coefficients.

Third, all data associated to gas flares are excluded from the nightlight database, because they are deemed to be irrelevant for the analysis, and finally, a 22-year time series of nightlights for each of the considered study regions is extracted.

\subsection{Preparation of Repurposed River Network Data and Identification of Distance Classes}

To identify how the human presence is settled across streams and rivers, five distinct distance-from-rivers classes are defined. The original vectorial CCM river network is converted to a raster file and then pixels are classified by adopting the euclidean distance approach. Distance-0 pixels represent river network pixels, distance-1 pixels identify all pixels adjacent to streams and rivers, while distance-2, distance- 3 , and distance- 4 pixels are defined from farther concentric zones (i.e., distance classes 1, 2, 3 and 4 correspond to 1, 2, 3 and $4 \mathrm{~km}$ far from the river network, respectively). 
Table 1. Nighttime lights data set, satellite number and observation year.

\begin{tabular}{ccccccc}
\hline & \multicolumn{5}{c}{ Satellite number } \\
\cline { 2 - 6 } & F10 & F12 & F14 & F15 & F16 & F18 \\
\hline 1992 & F101992 & & & & & \\
1993 & F101993 & & & & & \\
1994 & F101994 & F121994 & & & & \\
1995 & & F121995 & & & & \\
1996 & & F121996 & & & & \\
1997 & & F121997 & F141997 & & & \\
1998 & & F121998 & F141998 & & & \\
1999 & & F121999 & F141999 & & & \\
2000 & & & F142000 & F152000 & & \\
2001 & & & F142001 & F152001 & & \\
2002 & & & F142002 & F152002 & & \\
2003 & & & F142003 & F152003 & & \\
2004 & & & & F152004 & F162004 & \\
2005 & & & & F152005 & F162005 & \\
2006 & & & & F152006 & F162006 & \\
2007 & & & & F152007 & F162007 & \\
2008 & & & & & F162008 & \\
2009 & & & & & F162009 & \\
2010 & & & & & & F182010 \\
2011 & & & & & & F182011 \\
2012 & & & & & & F182013 \\
2013 & & & & & & \\
\hline
\end{tabular}

The performed analysis focuses on the following different groups of river pixels and associated distance-fromriver pixels (see Fig. 1): CCM river pixels with Strahler order (i) $\geq 1$ (i.e., all original river pixels are considered, where Strahler order $=1$ represents small streams); (ii) $\geq 2$; (iii) $\geq 3$; (iv) $\geq 4$ (Strahler order $=4$ identifies major rivers). Given that the CCM river network spatial resolution is more detailed than the nightlight one (i.e., 3 arcsec versus 30 arcsec), we decided to consider all these different categories of river pixels in order to examine how the human signatures, as derived from the spatio-temporal nightlight trends, are influenced by the river network definition. As a consequence, when the original CCM river network is considered, river pixels correspond to nearly $40 \%$ of the total number of pixels across Austria, Veneto and Friuli Venezia Giulia, while they are about $60 \%$ for Trentino Alto Adige (see Fig. 1). In the case of CCM river pixels with Strahler order $\geq 2, \geq 3$ and $\geq 4$, the associated river pixel percentage reduces to nearly $24 \%$ (for Austria, Veneto and Friuli Venezia Giulia) and 33\% (for Trentino Alto Adige), $13 \%$ (for Austria, Veneto and Friuli Venezia Giulia) and $17 \%$ (for Trentino Alto Adige) and, 7\% (for Austria, Trentino Alto Adige, Veneto and Friuli Venezia Giulia), respectively (see Fig. 1).

\subsection{Temporal and Spatial Distribution Analysis of Repurposed Nightlights for the Study Regions}

To account for the human presence as derived from nightlights, for each study region $x$ (Austria, Trentino Alto Adige, Veneto and Friuli Venezia Giulia) and for each year $t$ (from 1992 to 2013), we first analyse nocturnal light values gathered in correspondence of each of the five distance-fromriver classes $j$ (where $j$ ranges from 0 to 4): for each pixel $i$ laying on the selected distance class, a DN value for all considered years is obtained, labelled as $\operatorname{DN}_{i, j}(x, t)$.

In order to visualize tendencies in time, for each region $x$, for each year $t$ and for each distance-from-river class $j$, we evaluate the empirical frequency distribution of $\mathrm{DN}_{i, j}(x, t)$ values. In addition, we estimate a characteristic $\mathrm{DN}$ value, $\mathrm{DN}_{j}(x, t)$, by averaging values measured in pixels located along each distance class $j$ as:

$\mathrm{DN}_{j}(x, t)=\frac{\sum_{i=1}^{n_{j}} D N_{i, j}(x, t)}{n_{j}(x)}$,

where $n_{j}$ represents the pixel abundance for distance class $j$. Thus, regional average values are analysed from both a spatial and a temporal perspective to detect the possible presence of trends in space and time. 

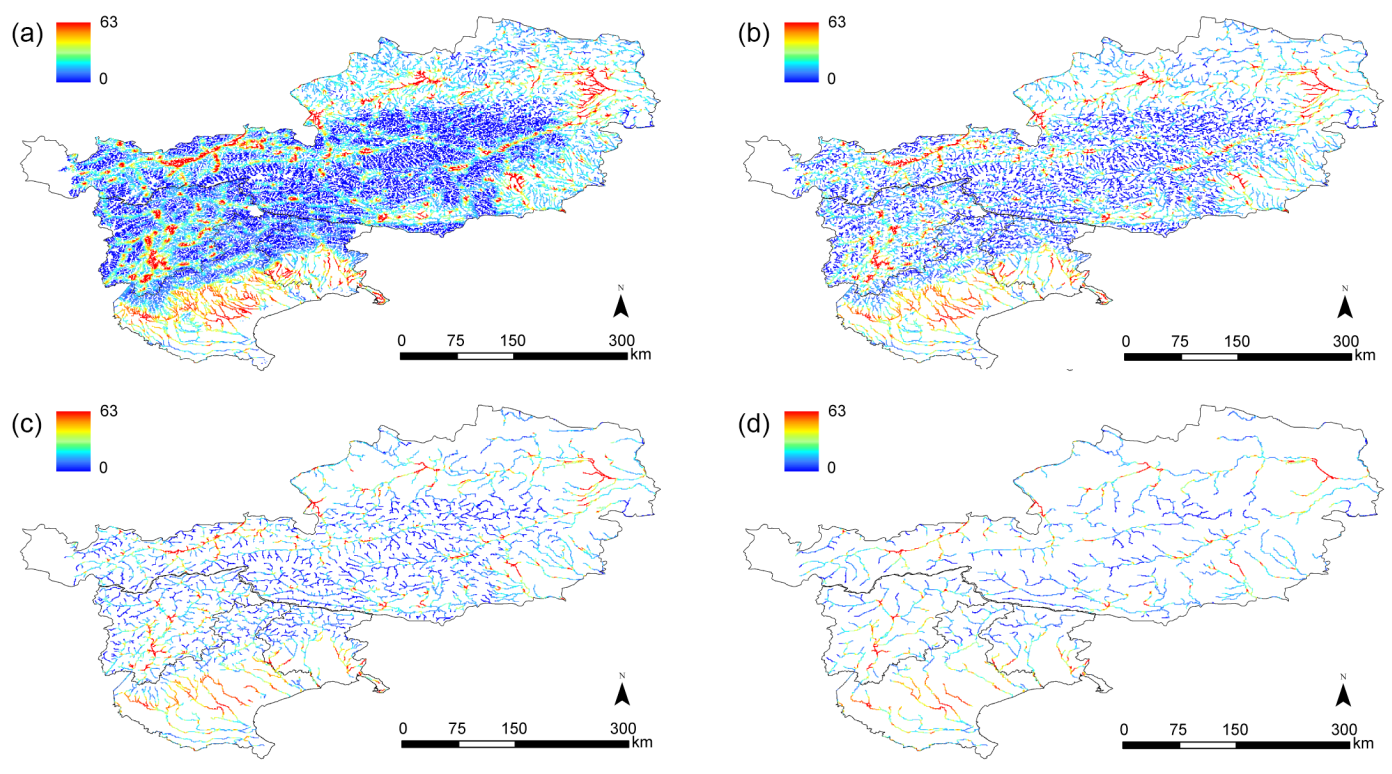

Figure 1. Repurposed nighttime lights (expressed as DN values) for year 2013 masked along the river network in the Eastern Alpine region (Austria and North Eastern Italian regions - Trentino Alto Adige, Veneto and Friuli Venezia Giulia): CCM river pixels with Strahler order (a) $\geq 1$; (b) $\geq 2$; (c) $\geq 3$ and (d) $\geq 4$ are considered.

\section{Results and discussion}

In order to identify human signatures across the European Eastern Alpine region, for each group of river pixels and associated distance-from-river pixels, we analysed repurposed nightlight data. Figure 1 shows, as an example, 2013 nightlights in correspondence of the river network pixels as defined from the aforementioned groups. As outlined in the legend, red pixels refer to areas with maximum DN values, which correspond to extensively urbanized areas (significant anthropogenic presence and associated economic activity), while blue pixels represent areas without nocturnal luminosity, likely characterized by the absence of human settlements and economic activities. Spatio-temporal trends of averaged nightlights $\mathrm{DN}_{j}(x, t)$ within each study region $x$ for all the considered distance-from-river classes $j$ (i.e., $j$ ranges from 0 to 4 ) and years $t$ (from 1992 to 2013) are then reported in Fig. 2.

From the temporal perspective, regardless of the group of river pixels identified by the Strahler order, a clear enhancement of nightlights in time is revealed for each study region and distance-from-river class, thus clearly proving that the human presence close to streams and rivers consistently increased in the Eastern Alpine region from 1992 to 2013.

From the spatial perspective, the nightlight trends across distance classes between the considered groups of river pixels reveal completely different behaviours. More specifically, river pixels with Strahler order $\geq 1$ and $\geq 2$ identify an extremely dense river network, which includes very small river reaches. Indeed, as shown in Fig. 1a, b, it seems that almost all pixels are streams or rivers. These two classes are domi- nated by upstream minor river reaches, which are likely not associated to human presence. Indeed, these pixels mainly lie in the natural environment of the Eastern Alps, not particularly influenced by anthropogenic activities. When moving far from the river network, average nightlights within these two classes tend to increase: this is valid for Austria, Veneto and Friuli Venezia Giulia, characterized by variable altitudes (including mountainous and plain areas), while it does not apply to Trentino Alto Adige, mainly constituted by mountains (Fig. 2, column 1 and 2).

Conversely, river pixels with Strahler order $\geq 3$ and $\geq 4$, which represent by definition only major streams and rivers, may provide a more reliable representation of human signatures. For these pixels, higher average nightlights are found for distance-0 pixels, while a decreasing luminosity trend is found in correspondence of higher distance-from-river classes (valid for all study regions, except for Strahler order $\geq 3$ in Friuli Venezia Giulia, see Fig. 2, column 3 and 4). These results confirm the main outcomes of Ceola et al. (2014, 2015), where the HydroSHEDS river network was adopted.

Concerning the ranges of variability of average $\mathrm{DN}_{j}(x, t)$ values, when comparing the different river network groups the following interesting features have emerged. For all the considered study regions, distance- 0 pixels present higher nocturnal luminosity values with increasing Strahler order river pixels. For Austria and Trentino Alto Adige, as the Strahler order increases, nightlights raise as well in all distance-from-river classes. In particular, while the lower DN values do not differ among the river network groups, there is an increment in the upper range DN value. Conversely, for 


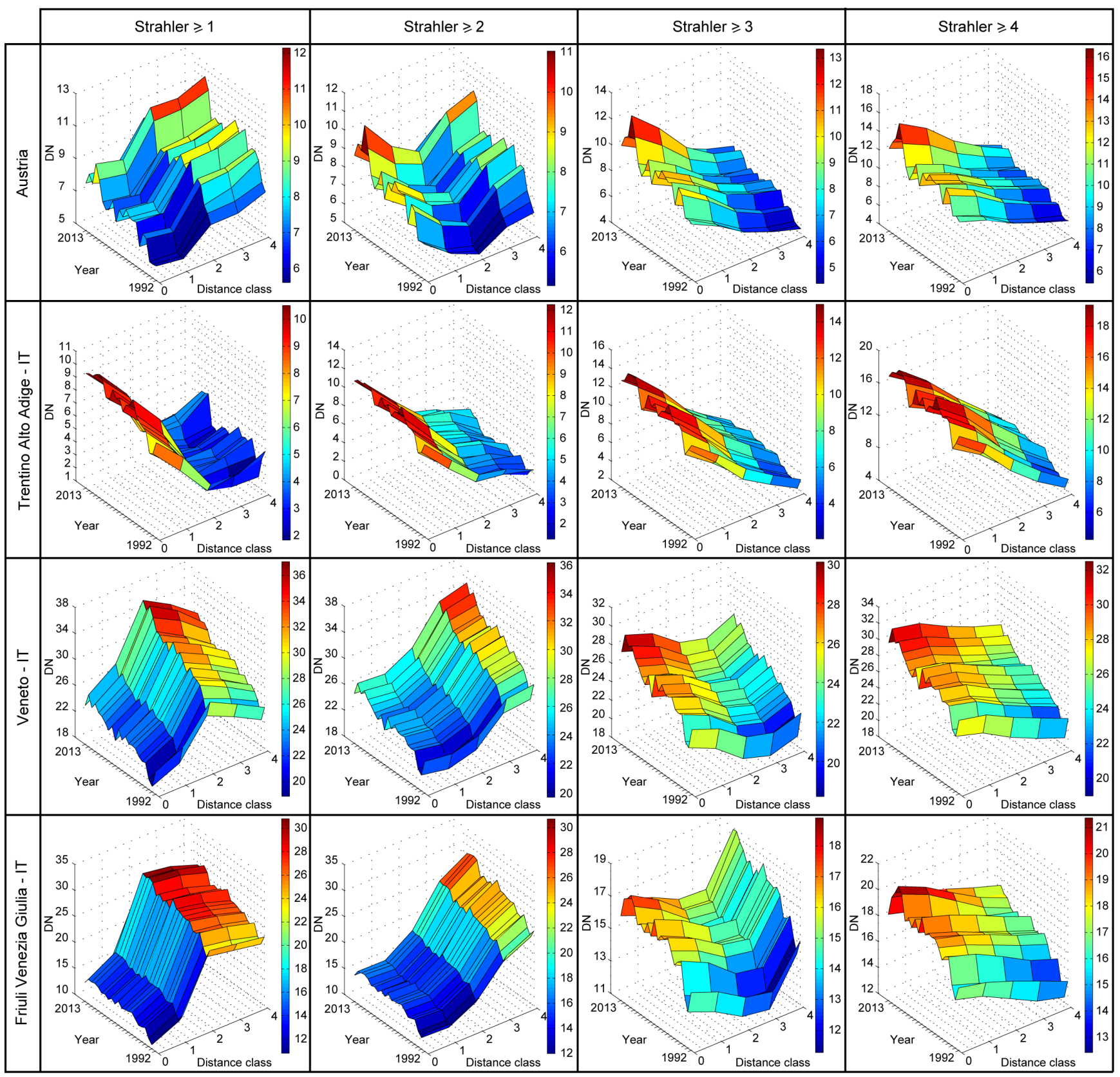

Figure 2. Spatial and temporal trend of yearly nightlight values in Austria (first row), Trentino Alto Adige (second row), Veneto (third row) and Friuli Venezia Giulia (forth row). Columns 1, 2, 3 and 4 refer to CCM river pixels with Strahler order $\geq 1, \geq 2, \geq 3$ and $\geq 4$, respectively. Distance classes $0,1,2,3$ and 4 correspond to: river pixels, 1, 2, 3 and $4 \mathrm{~km}$ far from the river network, respectively.

Veneto and Friuli Venezia Giulia, the maximum average luminosity evaluated for each distance class decreases with increasing Strahler order river pixels.

\section{Conclusions}

The spatially and temporally extensive analysis of human signatures from nightlights performed so far (i.e., by using detailed information on the river network location and nearby pixels) provide valuable insights into the evolution of human presence. Our results clearly point out an overall enhancement of human presence across the considered distance classes during the last 22 years, though presenting some differences among the study regions. From 1992 to 2013 average nightlights increased with respect to their initial value of about $30 \%$ for Austria, $15 \%$ for Trentino Alto Adige and $12 \%$ for Veneto and Friuli Venezia Giulia. In addition, the river network delineation is found to play a central role in the 
identification of nightlight spatio-temporal trends, as highlighted by our results. Perspective work envisages to compare these outcomes with demographic data, as well as the European Settlement Map (i.e., a spatial raster data set that is mapping human settlements in Europe based on SPOT5 and SPOT6 satellite imagery, produced with Global Human Settlement Layer technology by the European Commission, Joint Research Centre, Institute for the Protection and Security of the Citizen, Global Security and Crisis Management Unit). Furthermore, linkages between nightlights and drought issues due to enhanced human water demands are also planned to be uncovered.

Acknowledgements. The research leading to these results has received funding from the European Union Seventh Framework Programme (FP7/2007-2013) under grant agreement no. 603587, project "SWITCH-ON" (Sharing Water-related Information to Tackle Changes in the Hydrosphere - for Operational Needs).

\section{References}

Bennie, J., Davies, T. W., Duffy, J. P., Inger, R., and Gaston, K. J.: Contrasting trends in light pollution across Europe based on satellite observed night time lights, Sci. Rep., 4, 3789, doi:10.1038/srep03789, 2014.

Ceola, S., Laio, F., and Montanari, A.: Satellite nighttime lights revealing increased human exposure to floods worldwide, Geophys. Res. Lett., 41, 7184-7190, doi:10.1002/2014GL061859, 2014.
Ceola, S., Laio, F., and Montanari, A.: Human-impacted waters: New perspectives from global high resolution monitoring, Water Resour. Res., 51, 7064-7079, doi:10.1002/2015WR017482, 2015.

Chand, T., Badarinath, K., Elvidge, C., and Tuttle, B.: Spatial characterization of electrical power consumption patterns over India using temporal DMSP-OLS night-time satellite data, Int. J. Remote Sens., 30, 647-661, doi:10.1080/01431160802345685, 2009.

Chen, X. and Nordhaus W.: Using luminosity data as a proxy for economic statistics, P. Natl. Acad. Sci. USA, 108, 8589-8594, doi:10.1073/pnas.1017031108, 2011.

Elvidge, C., Baugh, K., Kihn, E., Kroehl, H., and Davis, E.: Mapping city lights with nighttime data from the DMSP operational linescan system, Photogramm. Eng. Rem. S., 63, 727-734, 1997.

Elvidge, C., Sutton, P., Ghosh, T., Tuttle, B., Baugh, K., Bhaduri, B., and Bright, E.: A global poverty map derived from satellite data, Comput. Geosci., 35, 1652-1660, doi:10.1016/j.cageo.2009.01.009, 2009.

Kummu, M., de Moel, H., Ward, P., and Varis, O.: How close do we live to water? A global analysis of population distance to freshwater bodies, PLoS ONE, 6, e20578, doi:10.1371/journal.pone.0020578, 2011.

Small, C.: Global population distribution and urban land use in geophysical parameter space, Earth Interact., 8, 1-18, 2004. 